OPEN ACCESS

Edited by:

Muthuraman Muthuraman,

Universitätsmedizin der Johannes

Gutenberg - Universität Mainz,

Germany

Reviewed by:

Giancarlo Zito,

Ospedale San Giovanni Calibita

Fatebenefratelli, Italy

Lidia Fernández Paredes,

Hospital Clínico San Carlos, Spain

*Correspondence:

Ole Pless

ole.pless@ime.fraunhofer.de

tThese authors have contributed equally to this work.

Received: 26 March 2018 Accepted: 15 May 2018

Published: 04 June 2018

Citation:

Stoessel D, Stellmann J-P, Willing A,

Behrens B, Rosenkranz SC,

Hodecker SC, Stürner KH, Reinhardt S, Fleischer S, Deuschle C,

Maetzler W, Berg D, Heesen C, Walther D, Schauer N, Friese MA and Pless O (2018) Metabolomic Profiles

for Primary Progressive Multiple Sclerosis Stratification and Disease Course Monitoring

Front. Hum. Neurosci. 12:226. doi: 10.3389/fnhum.2018.00226

\section{Metabolomic Profiles for Primary Progressive Multiple Sclerosis Stratification and Disease Course Monitoring}

Daniel Stoessel ${ }^{1,2,3}$, Jan-Patrick Stellmann ${ }^{4,5}$, Anne Willing $^{4}$, Birte Behrens ${ }^{6}$, Sina C. Rosenkranz ${ }^{4,5}$, Sibylle C. Hodecker ${ }^{4,5}$, Klarissa H. Stürner ${ }^{4,5}$, Stefanie Reinhardt ${ }^{4}$, Sabine Fleischer ${ }^{4}$, Christian Deuschle ${ }^{6}$, Walter Maetzler ${ }^{6,7}$, Daniela Berg ${ }^{6,7}$, Christoph Heesen ${ }^{4,5}$, Dirk Walther ${ }^{2,3}$, Nicolas Schauer ${ }^{1+}$, Manuel A. Friese ${ }^{4 t}$ and Ole Pless ${ }^{* *+}$

\footnotetext{
${ }^{1}$ Metabolomic Discoveries GmbH, Potsdam, Germany, ${ }^{2}$ Institut für Biochemie und Biologie, Universität Potsdam, Potsdam, Germany, ${ }^{3}$ Bioinformatik, Max-Planck-Institut für Molekulare Pflanzenphysiologie, Potsdam, Germany, ${ }^{4}$ Zentrum für Molekulare Neurobiologie Hamburg, Institut für Neuroimmunologie und Multiple Sklerose, Universitätsklinikum Hamburg-Eppendorf, Hamburg, Germany, ${ }^{5}$ Klinik und Poliklinik für Neurologie, Universitätsklinikum Hamburg-Eppendorf, Hamburg, Germany, ${ }^{6}$ Neurodegenerative Erkrankungen, Hertie-Institut für klinische Hirnforschung, Eberhardt-Karls-Universität Tübingen, Tübingen, Germany, ${ }^{7}$ Department of Neurology, Christian-Albrechts-Universität zu Kiel, Kiel, Germany, ${ }^{8}$ Fraunhofer IME ScreeningPort, Hamburg, Germany
}

Primary progressive multiple sclerosis (PPMS) shows a highly variable disease progression with poor prognosis and a characteristic accumulation of disabilities in patients. These hallmarks of PPMS make it difficult to diagnose and currently impossible to efficiently treat. This study aimed to identify plasma metabolite profiles that allow diagnosis of PPMS and its differentiation from the relapsing-remitting subtype (RRMS), primary neurodegenerative disease (Parkinson's disease, PD), and healthy controls ( $\mathrm{HCs}$ ) and that significantly change during the disease course and could serve as surrogate markers of multiple sclerosis (MS)-associated neurodegeneration over time. We applied untargeted high-resolution metabolomics to plasma samples to identify PPMS-specific signatures, validated our findings in independent sex- and age-matched PPMS and HC cohorts and built discriminatory models by partial least square discriminant analysis (PLS-DA). This signature was compared to sex- and agematched RRMS patients, to patients with PD and HC. Finally, we investigated these metabolites in a longitudinal cohort of PPMS patients over a 24-month period. PLSDA yielded predictive models for classification along with a set of 20 PPMS-specific informative metabolite markers. These metabolites suggest disease-specific alterations in glycerophospholipid and linoleic acid pathways. Notably, the glycerophospholipid LysoPC(20:0) significantly decreased during the observation period. These findings show potential for diagnosis and disease course monitoring, and might serve as biomarkers to assess treatment efficacy in future clinical trials for neuroprotective MS therapies.

Keywords: untargeted metabolomics, biomarker, PPMS, MS neurodegeneration, LysoPC(20:0) 


\section{INTRODUCTION}

Primary progressive multiple sclerosis (PPMS) affects a subgroup of multiple sclerosis (MS) patients and shows a highly variable disease progression with poor prognosis (Katz Sand, 2015). While patients rarely present with clinical relapses as present in the more common relapsing-remitting subtype (RRMS), progressive accumulation of disabilities is characteristic. These hallmarks of PPMS make it difficult to diagnose and treat (Ontaneda et al., 2017).

Diagnosis of MS is currently based on the revised McDonald criteria (Polman et al., 2011) that include clinical assessments and MRI. However, diagnosis, subtype stratification, or assessment of disease progression by imaging, clinical, and biological markers is not yet reliable (Tremlett et al., 2005; Koch et al., 2009; Stellmann et al., 2014). Due to their accessibility and overall stability, surrogate blood metabolite markers of neuronal injury could substantially improve our mechanistic understanding and our ability to quantify neurodegeneration in MS (Botas et al., 2015) and could be applied to neuroprotective treatment trials to assess efficacy (Fox et al., 2012; Friese et al., 2014). Untargeted metabolomics is a high-throughput technology that allows for simultaneous semi-quantitative measurements of various metabolite species in complex samples such as biological fluids (Patti et al., 2012). Therefore, this is a suitable technology to obtain a comprehensive view of the functional state of the human organism (Xiao et al., 2012; Want et al., 2013; Contrepois et al., 2015). In addition, the high sensitivity and reliability of this technology makes it suitable for monitoring metabolite changes over time (Floegel et al., 2011).

By analyzing blood plasma with high-resolution mass spectrometry (HRMS), our study set out to discover metabolic profiles specific for PPMS. We identified a panel of 20 metabolites which discriminated PPMS from RRMS, Parkinson's disease (PD), or healthy control (HC) and one metabolite which consistently decreased during a 24-month PPMS disease course.

\section{MATERIALS AND METHODS}

\section{Standard Protocol Approvals, Registrations, and Patient Consents}

All participants (healthy donors, patients, or guardians of patients) provided written informed consent and the study was approved by the local ethics committee (Board of Physicians, Hamburg, Nos. PV4405 and PV3961). The PD study was approved by the Ethics Committee of the Faculty of Medicine at the University of Tübingen (26/2007BO1 and 497/2009BO1).

\section{Patient Recruitment and Diagnosis}

Primary progressive multiple sclerosis $(n=33)$ and RRMS $(n=10)$ patients and HC $(n=33)$ were recruited at the University Medical Center Hamburg-Eppendorf. The participants underwent clinical assessment and provided plasma samples independent of meals. All HC in the study consist of both spouses and volunteers, which were recruited based on providing 'matched pairs' to patients with regard to age and sex. All HC underwent clinical assessment and reported no known autoimmune or neurological disease. All MS patients fulfilled the revised 2010 McDonald criteria (Polman et al., 2011) and had an Expanded Disability Status Scale (EDSS) below 7.0. RRMS patients, PPMS patients, and HC were recruited as a cross-sectional cohort, while additional PPMS patients were participating in a prospective observational longitudinal cohort study with annual visits and sampling of biomaterial. PPMS and RRMS patients have been off medication for at least three months. PD $(n=40)$ patients and HC $(n=20)$ were recruited at the Faculty of Medicine of the University of Tübingen, following equal procedures for blood withdrawal, processing, and storage as reported for the MS patients. PD diagnosis was based on the UK Brain Bank Society's criteria for PD (Hughes et al., 1992). Of note, 15 out of $40 \mathrm{PD}$ patients received levodopa (L-Dopa) treatment. Non-MS/PD-specific medication was absent, was not reported or was specific to single patients included in the study (one RRMS patient reported L-thyroxine supplementation). No patient or healthy donor reported diabetes as a comorbidity. The demographic and clinical features of patients with MS, $\mathrm{PD}$, and healthy controls (HCs) are summarized in Table $\mathbf{1}$. A detailed list of all participants investigated can be found in Supplementary Table S1. Inter-cohort age and gender (sex) dependencies have been determined by chi-square test (sex) and one-way ANOVA (age, disease duration (DD), and EDSS; Supplementary Table S2). Of note, all biosamples were collected between 8 and 12 am and donors were not fasted.

\section{Human Sample Preparation}

Peripheral blood was collected in S-Monovette ${ }^{\circledR} 9 \mathrm{ml}$, K3 EDTA, $92 \times 16 \mathrm{~mm}$ test tubes (Sarstedt, 02.1066.001) and centrifuged for $8 \mathrm{~min}$ at $1200 \times g$ at $4^{\circ} \mathrm{C}$. The supernatant was centrifuged at $4^{\circ} \mathrm{C}$ for an additional $10 \mathrm{~min}$ at $4300 \times g$. The supernatant of the second centrifugation step was aliquoted, shock frozen in liquid nitrogen and stored at $-80^{\circ} \mathrm{C}$ until further analysis.

\section{LC/MS and MS/MS Analysis}

The analysis workflow is summarized in Supplementary Figure S1. Plasma metabolites were extracted using $90 \%$ $\mathrm{MeOH}$ and $10 \%$ water spiked with internal standards with constant shaking for $15 \mathrm{~min}$ at $37^{\circ} \mathrm{C}(1000 \mathrm{rpm})$. Modified hydrophilic interaction chromatography (HILIC) was employed in combination with HRMS. Samples were pseudonymized twice and third-party concealment of the origin of respective specimens (HC or MS) was achieved by using uniquely coded vials. Samples were randomized on an Agilent 1290 UHPLC system (Agilent, Santa Clara, United States) with a ZIC-HILIC column $(10 \mathrm{~cm} \times 2.1 \mathrm{~mm}, 3 \mu \mathrm{m}$, Sequant, Merck) coupled to a high-resolution 6540 QTOF/MS detector (Agilent, Santa Clara, United States) operated in both positive and negative ESI mode in a detection range of $50-1700 \mathrm{~m} / z$ at $2 \mathrm{GHz}$ in an extended dynamic range. The LC solvent consisted of (A) $95 \% 10 \mathrm{mM}$ ammonium acetate with 5\% acetonitrile $\mathrm{pH} 6$ and (B) $95 \%$ acetonitrile with $5 \% 10 \mathrm{mM}$ ammonium acetate with a multi-step gradient (15 min runtime: $5 \%$ B from start to $1 \mathrm{~min}$, to $35 \% \mathrm{~B}$ at $8.5 \mathrm{~min}$, to $95 \% \mathrm{~B}$ at $9.5 \mathrm{~min}$, to 


\begin{tabular}{|c|c|c|c|c|c|c|c|c|}
\hline & \multicolumn{8}{|c|}{ Cross-sectional cohorts } \\
\hline & \multicolumn{2}{|c|}{ PPMS cohort A } & \multicolumn{2}{|c|}{ PPMS cohort B } & \multicolumn{2}{|c|}{ RRMS cohort } & \multicolumn{2}{|c|}{ Parkinson's cohort } \\
\hline & PPMS & $\mathrm{HC}$ & PPMS & $\mathrm{HC}$ & RRMS & $\mathrm{HC}$ & PD & $\mathrm{HC}$ \\
\hline Number $(n)$ & 13 & 13 & 20 & 20 & 10 & 10 & 40 & 20 \\
\hline Females ( $n, \%)$ & $8(61.5)$ & $7(70)$ & $7(35)$ & $7(35)$ & $7(70)$ & $7(70)$ & $16(60)$ & $13(65)$ \\
\hline Age (year $\pm s d)$ & $52.5 \pm 8.7$ & $51.8 \pm 8.7$ & $51 \pm 7.3$ & $51.3 \pm 6.7$ & $44.2 \pm 9.9$ & $48.0 \pm 10.0$ & $64.8 \pm 8.94$ & $65.9 \pm 7.13$ \\
\hline $\begin{array}{l}\text { Disease duration } \\
(\text { year } \pm s d)\end{array}$ & \multicolumn{2}{|c|}{$8.7 \pm 5.9$} & \multicolumn{2}{|c|}{$7 \pm 7.3$} & \multicolumn{2}{|c|}{$3.2 \pm 7.5$} & \multicolumn{2}{|c|}{$7.0 \pm 2.92$} \\
\hline $\begin{array}{l}\text { EDSS (mean } \pm s d \text { ), median } \\
\text { [range] }\end{array}$ & \multicolumn{2}{|c|}{$4.3 \pm 1.3,3.75[2.5-6.5]$} & \multicolumn{2}{|c|}{$3.7 \pm 1.3,3.5[2.0-6.0]$} & \multicolumn{2}{|c|}{$2.4 \pm 0.9,2.25[1.5-4.0]$} & - & - \\
\hline $\begin{array}{l}\text { T25FW (s, mean } \pm s d \text { ), } \\
\text { median [range] }\end{array}$ & \multicolumn{2}{|c|}{$6.5 \pm 1.8,5.7[4.5-9.4]$} & \multicolumn{2}{|c|}{$6.3 \pm 3,5.1[3.9-15.5]$} & \multicolumn{2}{|c|}{$4.9 \pm 1.8,4.15[3.3-8.8]$} & - & - \\
\hline $\begin{array}{l}\text { 6MWT ( } \mathrm{m}, \text { mean } \pm s d) \text {, } \\
\text { median [range] }\end{array}$ & \multicolumn{2}{|c|}{$356.6 \pm 108.8,375$ [230-530] } & \multicolumn{2}{|c|}{$407.3 \pm 107,407$ [180-590] } & \multicolumn{2}{|c|}{ n.a. } & - & - \\
\hline $\begin{array}{l}\text { SDMT (mean } \pm s d \text { ), } \\
\text { median [range] }\end{array}$ & \multicolumn{2}{|c|}{$-0.4 \pm 1.4,0[-3.0-1.5]$} & \multicolumn{2}{|c|}{$-0.8 \pm 1,-0.5[-3.0-0.5]$} & \multicolumn{2}{|c|}{$-0.4 \pm 1.3,0[-2.5-1.5]$} & \multicolumn{2}{|r|}{-} \\
\hline $\begin{array}{l}\text { Hoehn and Yahr stage } \\
\text { (mean } \pm \text { sd, [range]) }\end{array}$ & \multicolumn{2}{|c|}{-} & \multicolumn{2}{|c|}{-} & \multicolumn{2}{|c|}{-} & \multicolumn{2}{|c|}{$1.3 \pm 0.69,[1-3]$} \\
\hline $\begin{array}{l}\text { UPDRS total score } \\
\text { (mean } \pm s d, \text { [range]) }\end{array}$ & \multicolumn{2}{|c|}{-} & \multicolumn{2}{|c|}{-} & \multicolumn{2}{|c|}{-} & \multicolumn{2}{|c|}{$25.2 \pm 11.98,[6-57]$} \\
\hline
\end{tabular}

Longitudinal cohort (PPMS)

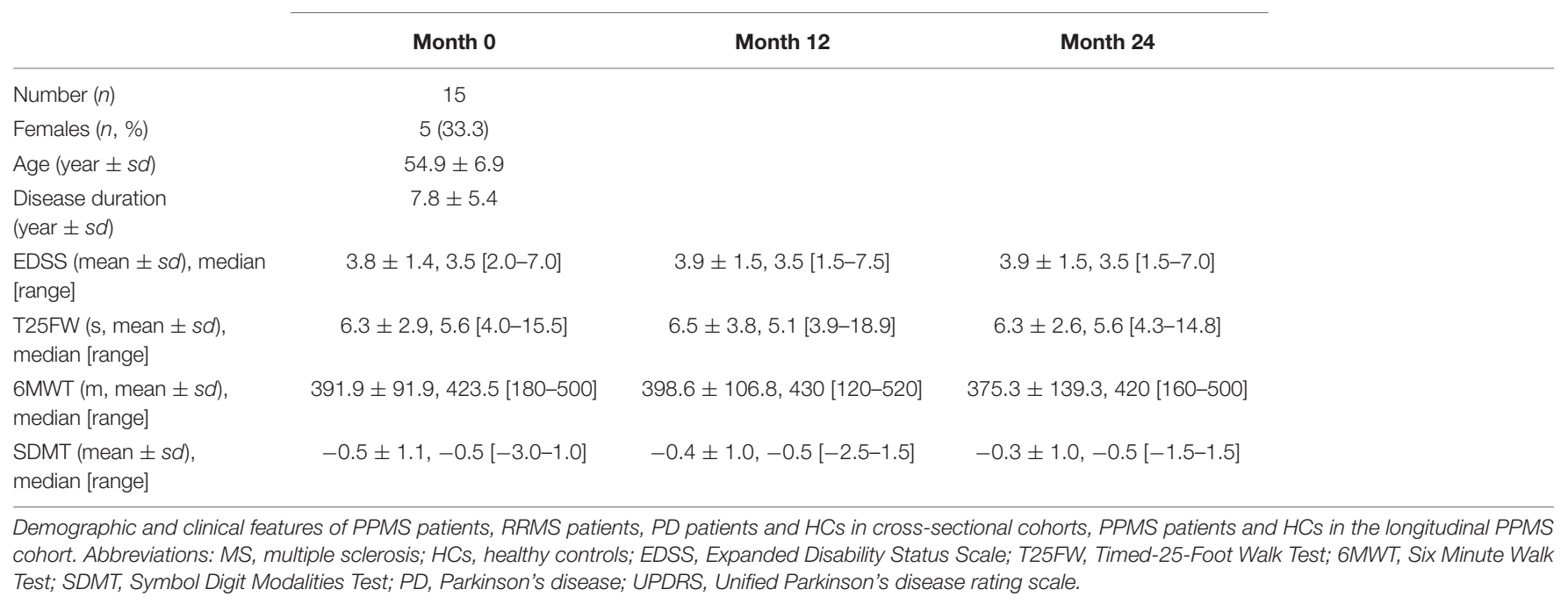

$5 \% \mathrm{~B}$ at $12.01 \mathrm{~min}$ until $15 \mathrm{~min}$ ). One microliter of sample was injected at $30^{\circ} \mathrm{C}$ column temperature and the flow rate was kept constant at $300 \mu \mathrm{l} / \mathrm{min}$. Chromatographic peaks, signal reproducibility, and analyte stability were monitored by assessment of biological quality controls, which were analyzed periodically throughout the batches. The DualAJS ESI source was set to the following parameters: Gas temperature $200^{\circ} \mathrm{C}$, drying gas $8 \mathrm{l} / \mathrm{min}$, nebulizer $35 \mathrm{psi}$, sheath gas temperature: $350^{\circ} \mathrm{C}$, sheath gas flow $11 \mathrm{l} / \mathrm{min}$, VCAp $3.5 \mathrm{kV}$, and nozzle voltage of $0 \mathrm{~V}$. Online calibration of the instrument was performed with the Agilent ESI-TOF Reference Mass Solution Kit. MS/MS spectra were acquired in positive and negative ionization modes in a data-dependent and targeted manner with fragmentation energies of $0,10,20$, and $40 \mathrm{~V}$, respectively. Precursor isolation windows varied between narrow $(1.3 \mathrm{~m} / z)$, medium $(4 \mathrm{~m} / z)$, and wide $(9 \mathrm{~m} / z$; performed with Agilent MassHunter software).

\section{Metabolomics Data Analysis}

Raw data were converted to mzXML and chromatogram peaks extracted with XCMS (Smith et al., 2006), which were optimized by using the IPO R-package (Libiseller et al., 2015). Mzmatch.R was used for peak filtering based on minimum detectable intensity (2000), peak shape filtering (codadw $>0.9$ ) and for the annotation of related peaks (Scheltema et al., 2011). Additional filtering was performed by excluding peaks with lower median peak intensities per group in biological samples compared to blanks (extraction solvent only). The remaining data were normalized based on multiple internal standards applying Normalization using Optimal selection of Multiple 
Internal Standards (NOMIS; Sysi-Aho et al., 2007) and CrossContribution compensating Multiple standard Normalization (CCMN; Redestig et al., 2009) normalization. IDEOM software was used $^{1}$ (Creek et al., 2012) to eliminate noise and artifacts and for putative peak annotation by exact mass within $\pm 10 \mathrm{ppm}$ against the Metabolomic Discoveries (MD) in-house metabolite library in negative and positive ESI modes, respectively. The MD metabolite database consists of $\sim 100 \mathrm{k}$ metabolites from various classes such as lipids, peptides, and amino acids. Metabolite identification was performed as previously reported (Stoessel et al., 2018). Briefly, to aid metabolite annotation, retention time prediction was applied (Creek et al., 2011). Identities were confirmed by available authentic standards (validation level 1) and MS/MS spectra matched with online databases (e.g., Metlin and MassBank; validation level 2) or with in silico fragmentation spectra (validation level 3) from Metfrag (Ruttkies et al., 2016), CFM-ID (Allen et al., 2014), and/or CSI:FingerID (Duhrkop et al., 2015). Precursor mass accuracy was set to $20 \mathrm{ppm}$ and fragment accuracy to $0.01 \mathrm{Da}$. Quantification of each metabolite was calculated using the raw peak height. Respective MSMS fragments were used for peak identification (Supplementary Table S3). Extracted ion chromatograms (EICs) of the determined PPMS markers can be found in Supplementary Figure S2. To allow for inter cohort comparisons, different run days have been normalized to zero mean differences between biological quality controls for peak intensities of the PPMS marker panel. These quality controls were injected before every batch.

\section{Statistical Analysis}

Univariate statistical analyses utilized a Welch's $t$-test $(p=0.05)$ and multivariate analyses utilized the metabolomics $\mathrm{R}$ package (De Livera and Bowne, 2014). Univariate area under the curve (AUC) measures and 95\% confidence intervals (CIs) using 500 bootstrappings were calculated utilizing MetaboAnalyst (Xia et al., 2015) and Rmisc R package (Hope, 2013). Class discrimination and membership prediction was performed for subgroups of PPMS cohorts A and B by using 70\% of all individuals for model training and the remaining $30 \%$ for blinded model testing. To ensure reproducibility of these results, patients were randomly selected for model testing and training 100 times. Additional class discrimination and membership prediction was performed by training a four-component PLS-DA model with all individuals from the PPMS cohort A and validating it in the blinded PPMS cohort B. Performances were gauged by the measures AUC, positive predictive values (PPVs), negative predictive values (NPVs), $R^{2} X / R^{2} Y$ (fraction of the variation of the $X / Y$ variables explained by the mode), $Q^{2}$ (cum), and accuracy of the calculated model. The datasets were scaled (zero mean, unit variance) prior to model building. For model prediction and AUC analysis, R-packages ROCR (Sing et al., 2005) and caret (Kuhn et al., 2016) were used. Furthermore, 'variable importance in the projection' (VIP) scores were computed and Welch's $t$-test was applied to determine discriminatory variables in the dataset. The VIP is an estimation of the importance of each variable

${ }^{1}$ http://mzmatch.sourceforge.net/ideom.php in the projection used in the partial least square discriminant analysis (PLS-DA) model as a quantitative estimation of the discriminatory power of each individual feature. Variables with a VIP score of $\geq 1$ were considered important in the PLS-DA model. To investigate for potential confounding factors, these putative markers were tested for sex dependencies (Welch's $t$-test) in PPMS cohort A and PPMS cohort B, and for age dependencies in PPMS cohort A, PPMS cohort B, RRMS cohort, and the $\mathrm{PD}$ cohort by linear regression. Moreover, to test whether these metabolites were able to discriminate PPMS from RRMS and PD, all PPMS patients and PD patients were combined. For the comparison of PPMS vs. RRMS, all individuals of the PPMS cohort A were used in combination with all RRMS patients to balance for class differences and potential gender dependencies. PLS-DA models were built using a random split of $70 \%$ of all individuals for model training and the remaining $30 \%$ for blinded model testing for each comparison. Again, to ensure reproducibility of these results, this random split was performed 100 times. To ensure that the obtained AUC, PPV, and NPV and accuracy values were higher than by chance, subject labels were randomly shuffled 100 times in the comparisons PPMS cohort A vs. HC, PPMS cohort B vs. HC, PPMS cohort A combined with PPMS cohort B vs. PD and PPMS cohort A vs. RRMS and empirical $p$-values were calculated. Univariate $p$-values for the comparison of PPMS vs. PD vs. RRMS were obtained using oneway ANOVA followed by Tukey's post hoc test, which includes $p$-value adjustment for multiple comparisons. Additional $p$-value adjustments for multiple metabolites were carried out by using Benjamini and Hochberg (1995) false discovery rate (FDR) adjustment. Moreover, all $p$-values from paired and unpaired Welch's $t$-tests were corrected for multiple testing (FDR).

Partial least square discriminant analysis computations were performed using the mixOmics (Le Cao et al., 2015) and ROPLS R-package (Le Cao et al., 2009; Thevenot et al., 2015). Suitable markers were selected, which enabled discrimination between $\mathrm{HC}$ and PPMS patients in the longitudinal cohort and time points 0 month (baseline), 12 months and 24 months utilizing paired $t$-test statistics $(p<0.01)$. These levels were also compared to levels of HC from our PPMS cohorts and RRMS and PD patients using one-way ANOVA followed by Tukey's post hoc test. Significant levels in MetaboAnalyst pathway analysis were based on hypergeometric tests and the pathway impact values determined by relative-betweenness centrality (Xia et al., 2015).

\section{RESULTS}

\section{Plasma Metabolites Distinguish PPMS Patients From Healthy Controls}

In order to identify metabolites that differentiate PPMS from HCs, we first employed a comparative untargeted metabolomics approach in plasma of HC $(n=33)$ and PPMS patients $(n=33)$, which we separated in two cohorts, cohort A $(n=13$ per group, exploration cohort) and cohort B $(n=20$ per group, validation cohort) (Supplementary Figure S1A). After individual measurements, we combined peak extraction procedures on the raw data for PPMS cohorts A and B to maximize the amount 
of overlapping mass peaks. Overall, 19,233 peaks in positive and 10,805 peaks in negative ionization modes were present in PPMS cohorts A and B. The within experiment technical and analytical variations were monitored based on periodic analysis of biological quality control samples in all cohorts. The median relative standard deviation (RSD) as an indicator for analytical reproducibility was $<10 \%$, which is within acceptable limits for metabolomics (Kirwan et al., 2014). Successive noise filtering, putative peak annotation, and combination of both ionization modes resulted in nomination of 534 putative metabolites (Supplementary Table S4). Identified metabolite classes and their prevalence are summarized in Supplementary Figure S1B. We analyzed the data by a supervised, multivariate classification technique (PLS-DA) to separately assess the overall segregation of the samples for PPMS cohorts A and B. Notably, this analysis allowed us to reproducibly separate HC from PPMS samples in both cross-sectional PPMS cohorts (Supplementary Figures S1C,D). In addition to this global and exploratory analysis that included all individuals, we tested whether the measured metabolites had a robust predictive value. Cohorts were split into training and test sets for deriving a PLS-DAbased model and to test its predictive performance, respectively (see section "Materials and Methods" for details). We used receiver operating characteristics (ROC) analysis of the training and testing model and determined a mean AUC of $79 \%(95 \%$ $\mathrm{CI}=76-82)$, a mean PPV of $0.75(95 \% \mathrm{CI}=0.72-0.78)$, and a mean NPV of $0.84(95 \% \mathrm{CI}=0.80-0.88)$ for cohort A. In our PPMS cohort B, we could determine a mean AUC of $73 \%$ $(95 \% \mathrm{CI}=72-74)$, a mean PPV of 0.69 (95\% CI $=0.66-0.72)$, and a mean NPV of 0.70 (95\% CI $=0.66-0.73)$. In addition, we used a PLS-DA model trained on PPMS cohort B data including all subjects to predict class memberships of the blinded PPMS cohort A. This cross-cohort test yielded an overall AUC of 70\% (95\% CI $=48-80)$, accuracy of 0.65 , PPV of 0.65 , and NPV of 0.65 (Figure 1). Random shuffling of subject labels in each cohort in this cross-cohort test indicates that the obtained model parameters were significantly larger than expected by chance ( $p$-value $\leq 0.05)$. Detailed parameters for all tested models can be found in Supplementary Table S5.

\section{PPMS Patients Differentiate From Healthy Controls, RRMS, and PD Patients}

Based on the PLS-DA models for each PPMS cohort, we extracted metabolites that contributed significantly to the differentiation between PPMS and $\mathrm{HC}$ in cohort A $\left(R^{2} X=0.24, R^{2} Y=0.98\right.$, $Q^{2}($ cum $\left.)=0.68\right)$ and cohort $\mathrm{B}\left(R^{2} X=0.09, R^{2} Y=0.86\right.$, $Q^{2}($ cum $\left.)=0.45\right)$ by using VIP scores as a quantitative estimation of the discriminatory power of each individual metabolite. VIP scores were extracted for components 1 and 2 since they separated HC and PPMS almost to the same extent (Supplementary Figures S1C,D). Overall, 20 metabolites with a VIP score greater than 1 were reproducibly determined in both the PPMS cohorts A and B (11\% of all metabolites with VIP $\geq 1$ in PPMS cohort A and 12\% of all metabolites with VIP $\geq 1$ in PPMS cohort B) with univariate AUC values $\geq 60 \%$ (Table 2

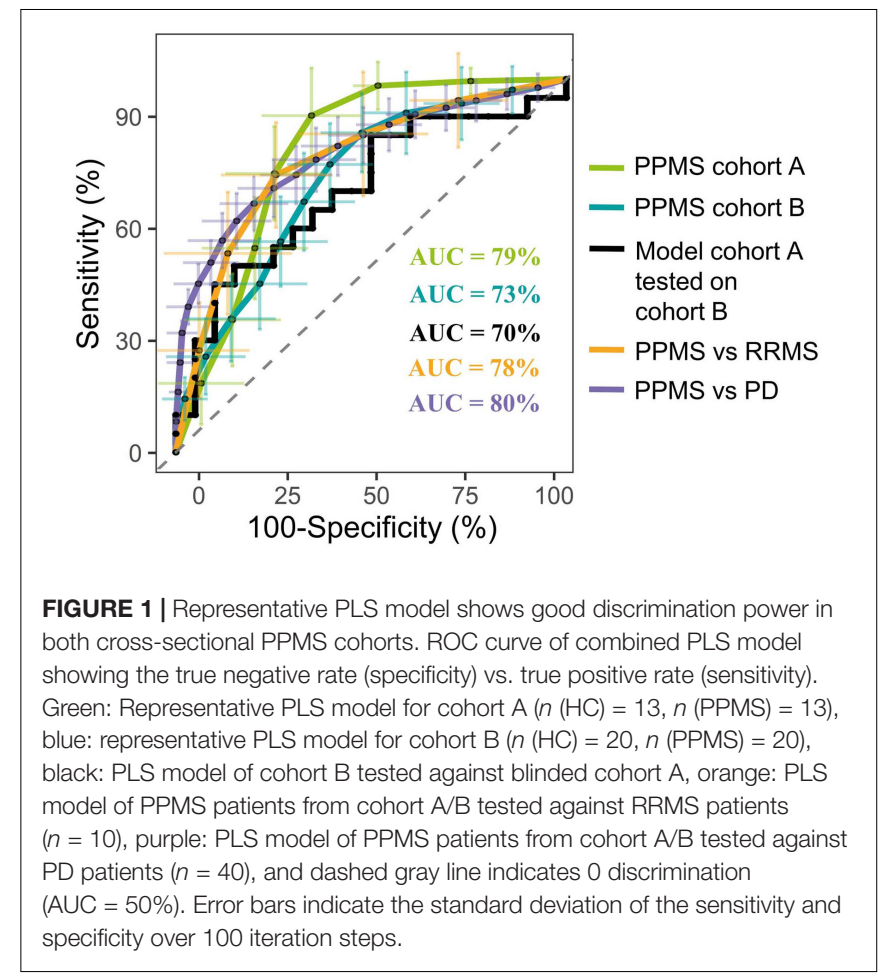

and Supplementary Table S6). Random shuffling of subject labels in each cohort yielded an empirical value of $p=0.004$ for the number of 20 commonly found informative metabolites indicating that the overlap between cohorts is significantly larger than expected by chance. Of note, all of these metabolites were diminished in PPMS patients compared to HC and several were also significantly altered in either one or both PPMS cohorts (Welch's $t$-test FDR $\leq 0.05$; Figure $2 \mathrm{~A}$ ). To determine the specificity of these 20 metabolites for PPMS, we measured these metabolites in an independent cohort of 10 RRMS patients and $10 \mathrm{HC}$ and an independent cohort of $40 \mathrm{PD}$ patients and $20 \mathrm{HC}$. In our inter-disease comparison, we found levels of LysoPE(18:2) and LysoPC(20:0) to be significantly lower in PPMS compared to RRMS and PD (one-way ANOVA $p \leq 0.05$ with Tukey's post hoc test). Moreover, tiglylcarnitine was found to be significantly higher in PPMS compared to RRMS (one-way ANOVA $p \leq 0.05$ with Tukey's post hoc test; Figure 2B and Supplementary Table S7). Of note, these differences appeared to be only significant (FDR $\leq 0.05$ ), when $p$-value correction was performed for comparison of multiple groups. Compared to $\mathrm{HC}$, we found some of the metabolites to be consistently downregulated in all diseases analyzed, indicating a general signature of neurodegeneration, but also found clear diseasespecific differences, e.g., a downregulation of the metabolites gamma-Linolenic acid, (L)-tryptophan, and LysoPC(20:0) in PPMS and an upregulation of these analytes in RRMS and PD (Figure 2B and Supplementary Tables S8, S9).

Multivariate analysis of these 20 metabolites for the discrimination compared to RRMS $\left(R^{2} X=0.79, R^{2} Y=0.93\right.$, $\left.Q^{2}(\mathrm{cum})=0.93\right)$ led to a mean AUC of $78 \%(95 \% \mathrm{CI}=75-82)$, mean PPV of $0.72(95 \% \mathrm{CI}=0.68-0.76)$, mean NPV of 0.77 


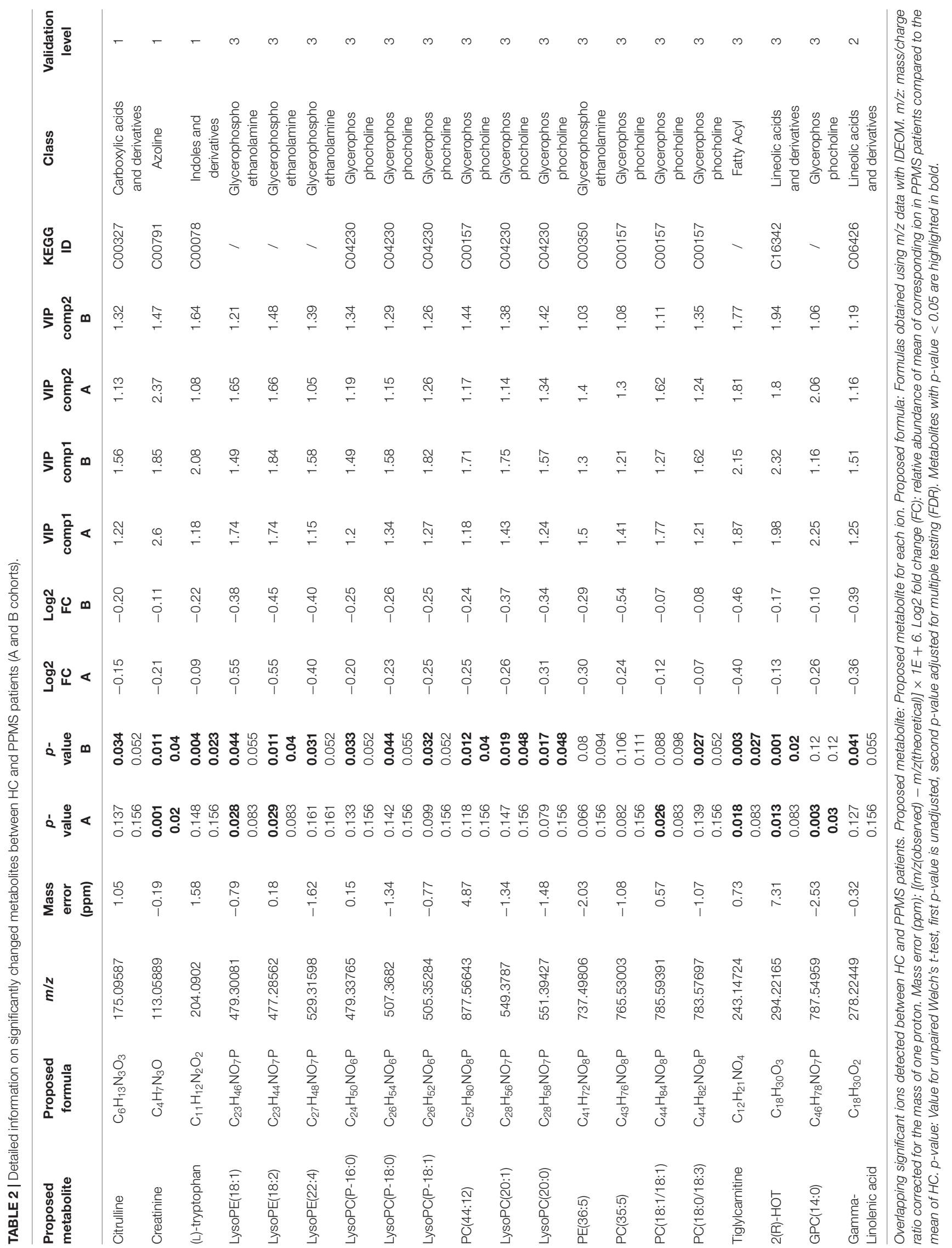




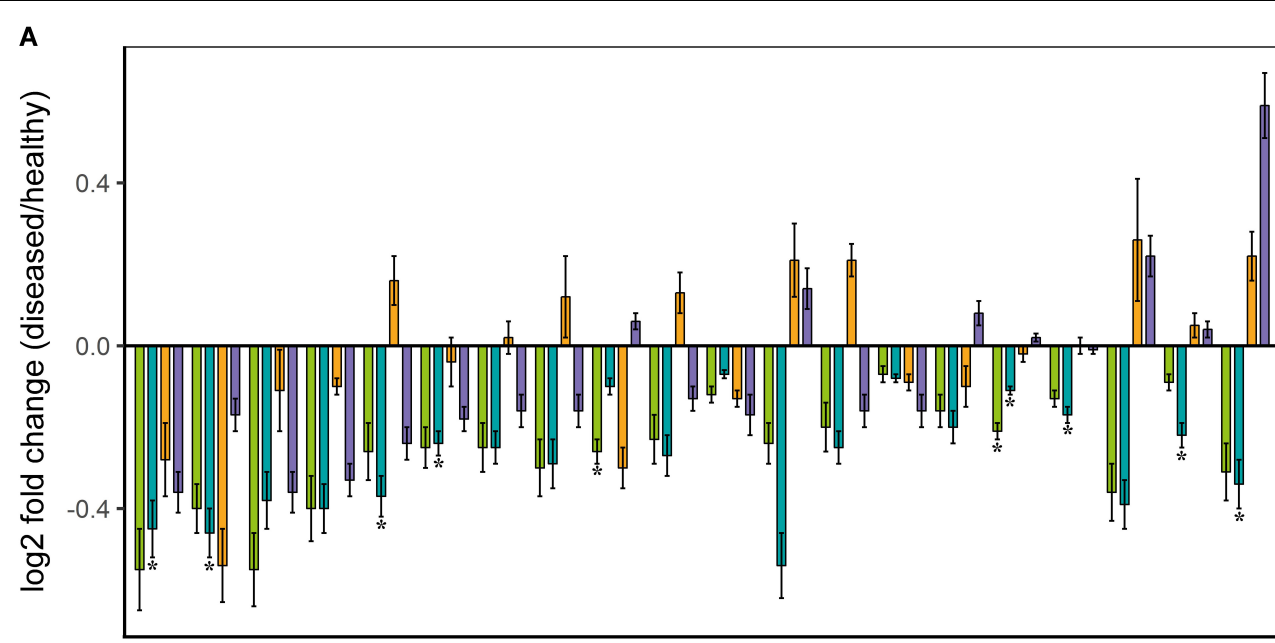

B

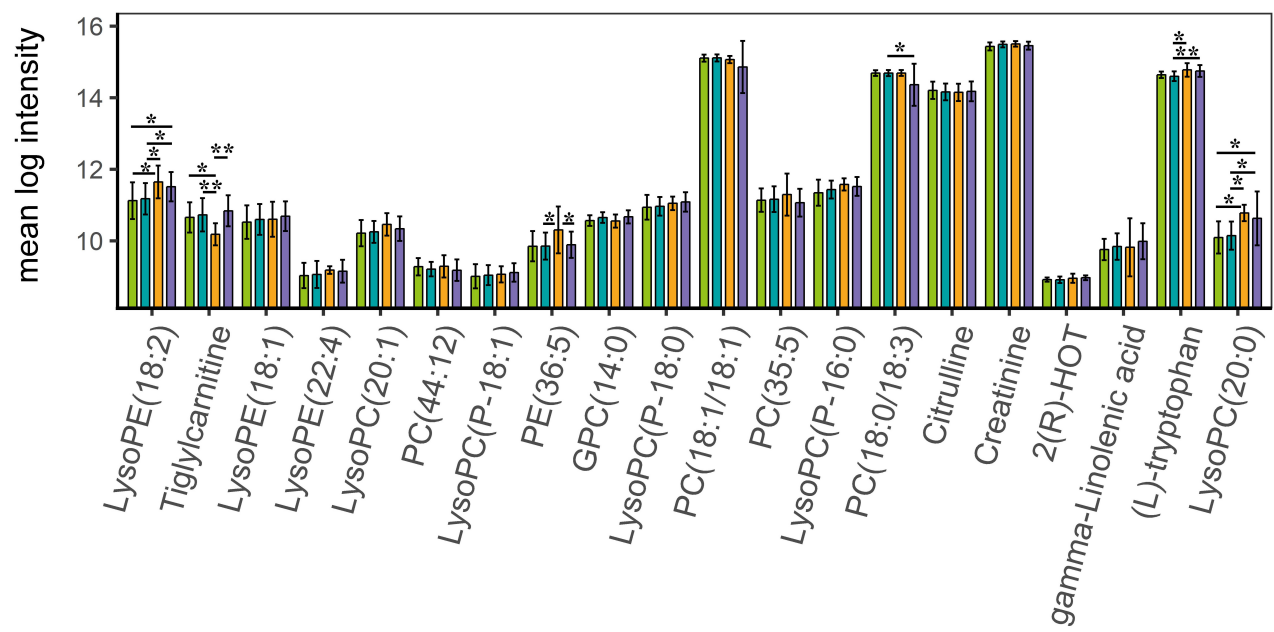

PPMS cohort A PPMS cohort B RRMS PD

FIGURE 2 | Metabolites contributing to the specific PPMS signature. Twenty features (i.e., metabolites) with VIP scores > 1 for components 1 and 2. (A) Summary with respective log2 fold changes (diseased/healthy). Green: PPMS cohort A, blue: PPMS cohort B, orange: RRMS, and purple: PD. *Significant change (Welch's $t$-test FDR $\leq 0.05$ ), log2 fold change $>0$ : upregulation, log2 fold change < 0: downregulation. Error bars indicate standard deviations. (B) Comparison of PPMS patients from cohort A (green) and PPMS patients from cohort B (blue) to RRMS (orange) and PD (purple) patients. *Significant change (one-way ANOVA with Tukey's post hoc test $p \leq 0.05$ (** $p \leq 0.01$ ), $p$-value adjusted for multiple comparisons).

$(95 \%$ CI $=0.73-0.81)$, and a mean accuracy of $0.68(95 \%$ $\mathrm{CI}=0.65-0.71)$. Multivariate discrimination relative to $\mathrm{PD}$ $\left(R^{2} X=0.69, R^{2} Y=0.56, Q^{2}(\mathrm{cum})=0.56\right)$ to a mean $\mathrm{AUC}$ of $80 \%(95 \% \mathrm{CI}=78-82)$, mean PPV of 0.75 (95\% CI $=0.73-0.77)$, mean NPV of $0.71(95 \% \mathrm{CI}=0.68-0.73)$, and a mean accuracy of 0.71 (95\% CI = 0.70-0.73; Figure 1). Again, random shuffling of subject labels in each comparison indicates that the obtained model parameters are significantly larger than expected by chance $(p \leq 0.05)$. Overall, the identified markers indicate a PPMS-specific plasma signature.

\section{PPMS-Specific Metabolites Profile Suggest Alterations in Glycerophospholipid Metabolism}

Based on human metabolome database (HMDB; Wishart et al., 2013) and KEGG identifiers of the 20 altered PPMS-specific metabolites, we set out to perform pathway analysis utilizing MetaboAnalyst (Xia et al., 2015). Overall, our untargeted metabolic profiling revealed several significant perturbations, which allowed identification of multiple significant biochemical pathways in particular in the glycerophospholipid pathway and linoleic acid metabolism (FDR $\leq 0.05$; Figure 3$)$.

\section{Levels of LysoPC(20:0) Decline During the Disease Course of PPMS}

Finally, we investigated the ability of the determined metabolite profile to monitor disease course by analyzing a longitudinal PPMS cohort $(n=15)$ over 24 months. Patients were clinically investigated and their plasma was sampled at baseline, after 12 and 24 months. Notably, 18 of 20 determined metabolites showed no significant change between baseline ( 0 months), 12 months, and 24 months disease course (paired $t$-test 


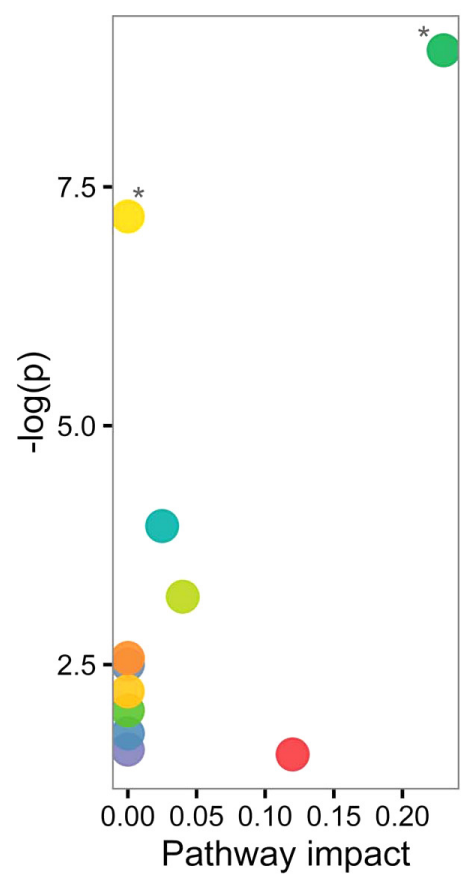

Pathway

Alpha-linolenic acid metabolism

Aminoacyl-tRNA biosynthesis

Arachidonic acid metabolism

Arginine and proline metabolism

Glycerophospholipid metabolism

Glycine, serine and threonine metabolism

Glycosylphosphatidylinositol(GPI)-anchor biosynthesis

Linoleic acid metabolism

Nitrogen metabolism

Phenylalanine, tyrosine and tryptophan biosynthesis

Tryptophan metabolism

FIGURE 3 | Pathway analysis of altered metabolites using MetaboAnalyst shows perturbations in glycerophospholipid and linoleic acid pathways. Twenty significantly deregulated metabolites identified were subjected to MetaboAnalyst (Xia et al., 2015) to assess association of each metabolite with defined pathways. $X$-axis presents pathway impact values (based on relative-betweenness centrality) and the $Y$-axis presents the respective $p$-values (based on hypergeometric test). ${ }^{*} p$-value $\leq 0.05$ after FDR correction. The largest and significant impact was found in glycerophospholipid and linoleic acid pathways.

$p<0.01)$. However, the glycerophospholipid annotated as LysoPC(20:0) showed a significant reduction when comparing 12 months and 24 months values ( $p$-value $=0.0034$, FDRadjusted $p$-value $=0.0576$; paired $t$-test $)$, fold change $(12$ months $/ 24$ months $)=1.59)$ or baseline $(0$ month $)$ and 24 months values $(p$-value $=0.0044$, FDR-adjusted $p$-value $=0.0888$ (paired $t$-test), fold change $(0$ month $/ 24$ months $)=1.84$; Figure 4 and Supplementary Table S10). Therefore, our data show a strong association of LysoPC(20:0) with the PPMS disease course over time. Moreover, LysoPC(20:0) baseline values were found significantly lower compared to $\mathrm{HC}$ in PPMS cohort A ( $p$-value $=0.0316), \mathrm{HC}$ in PPMS cohort $\mathrm{B}$ $(p$-value $=0.0036)$, RRMS patients $\left(p\right.$-value $\left.=7.27 \times 10^{-5}\right)$, and $\mathrm{PD}$ patients $\left(p\right.$-value $=1.49 \times 10^{-6}$, one-way ANOVA and Tukey's post hoc test). Since none of the recorded clinical measures (EDSS, SDMT, and walking tests) showed a significant change over the investigated time of the PPMS disease course (ANOVA $p$-value $\leq 0.05$ ), we were restricted in performing further correlative analysis. Recent evidence, however, suggests that significant changes in clinical parameters are unlikely to manifest during a 24-month time period in PPMS (Signori et al., 2017). We could not find a correlation between LysoPC(20:0) levels and corresponding patient's age (linear regression, $p$-value $=0.22$ ).

\section{Analysis of Confounding Factors}

A number of tests were performed to rule out confounding factors in our analysis. We reduced the sex bias in MS (Voskuhl and Gold, 2012) by comparing equal sex distributions in the different cohorts (HC, PPMS, RRMS, PD, and longitudinal cohort). In addition, we compared strictly age-matched cohorts. RRMS patients are on average younger than PPMS patients and the cohorts analyzed in this study represent 'real life' patients who visit our outpatient clinics. Nevertheless, the age was not significantly different between PPMS cohorts A and $B$ and the RRMS cohort (Supplementary Table S8). Therefore, we decided not to normalize for age differences between these two cohorts. Furthermore, we tested for an age-related bias in the analysis, which we could refute for LysoPC(20:0) (age difference not significant according to ANOVA, $p \leq 0.05$, Supplementary Figure S3). Furthermore, age-related dependencies could only be identified for gammaLinolenic acid (negative correlation) and LysoPC(20:1) (positive correlation; Supplementary Figure S3). We also tested for any sex-related bias in this analysis. Overall, several metabolites appeared to show significantly (ANOVA, $p \leq 0.05$ with Tukey's post hoc test) different levels between males and females in PPMS cohorts A and B (Supplementary Figure S4). To compare equal gender distributions among groups for our PPMS vs. RRMS comparison, we used PPMS patients from PPMS cohort A when tested for specificity against RRMS patients. Since the individuals in our PD cohort were significantly older (ANOVA $p \leq 0.05$ ) than our PPMS patients, we also tested for potential age-related trends of the identified 20 metabolites in the PD cohort. We only observed significant negative correlations (linear model $p<0.05$ ) for gamma-Linolenic acid and (L)-tryptophan 

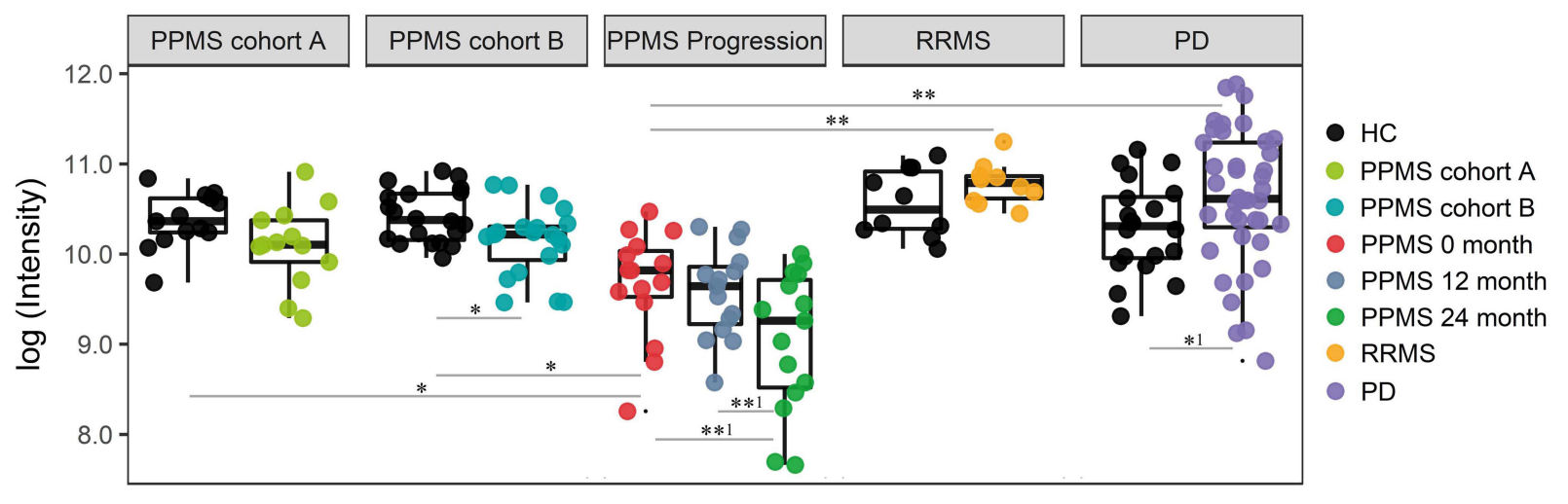

FIGURE 4 | Intensity levels for glycerophospholipid LysoPC(20:0) show relative differences in abundance in patients during PPMS disease course. PPMS cohort A: Decreased LysoPC(20:0) levels in PPMS patients compared to HC, black: HC $(n=13)$, green: PPMS patients $(n=13)$. PPMS cohort B: LysoPC(20:0) levels lower in PPMS patients compared to HC, black: HC $(n=20)$, blue: PPMS patients $(n=20)$. PPMS progression: Data from the disease course cohort showing decreasing levels of LysoPC(20:0) (0, 12, and 24 months: $n=15)$, red: baseline (0 month), dark blue: PPMS 12 months, and green: PPMS 24 months. RRMS: Black: HC $(n=10)$, yellow: RRMS patients $(n=10) . P D$ : black: HC $(n=20)$, purple: PD patients $(n=40)$. LysoPC(20:0) is significantly elevated in PD and elevated in RRMS patients compared to HC. ${ }^{*} \mathrm{FDR} \leq 0.05,{ }^{*} \mathrm{FDR} \leq 0.01,{ }^{* * 1} \mathrm{FDR} \leq 0.1,{ }^{* 1} p$-value $\leq 0.05$ without FDR correction.

(Supplementary Figure S5). Since these metabolites do not show any significant differences between PPMS and PD, we decided not to normalize for these effects. In addition, we could not identify any significant age-related trends in the RRMS cohort (Supplementary Figure S6). Finally, even though 15 out of 40 PD patients received L-Dopa, this treatment showed no significant effect when compared to treatment-naive PD patients on any of our 20 PPMS metabolites (data not shown, Welch's $t$-test $p$-value $\leq 0.05$ ).

\section{DISCUSSION}

\section{PPMS Metabolite Panel Discriminates From HC, RRMS, and PD}

Primary progressive multiple sclerosis is a clinically highly variable inflammatory neurodegenerative disease and is associated with a poor prognosis and continuous accumulation of neurological symptoms and disabilities (Antel et al., 2012). Currently, no reliable molecular biomarker-based diagnosis or monitoring of disease course is available. These would, however, be of utmost importance for the development of novel therapeutic options tackling PPMS-associated neurodegeneration.

Untargeted metabolomics allows for the simultaneous identification and quantification of a wide range of metabolites in biofluid samples which can be used to differentiate between 'healthy' and 'diseased' and can lead to the discovery of pathways involved in pathogenesis. The generated PLS-DA models showed a moderate discriminatory power to segregate PPMS patients and healthy individuals. The PLS-DA model trained with PPMS cohort B was tested on blinded cohort A and achieved an AUC of $70 \%$. While significantly better than randomly expected, also corroborated by the significant correlation of metabolite VIP scores between the two cohorts (Figure 1), this performance does not yet allow to conclude general diagnostic utility of the PLS model. Aside from the limitations in set size, this may be indicative of unique metabolic signatures in each cross-sectional PPMS cohort most likely caused by cohort effects such as differences in sample storage duration and physiological variance between individuals. Also, performing sampling at defined time points of the day, preferably from fasted individuals, would have been optimal for this study but is difficult to achieve in clinical practice. Nonetheless, a subset of 20 significantly altered metabolites was determined reproducibly with a good separation between the investigated PPMS patients and HC, RRMS, and PD. Thus, they can be considered PPMS specific.

A potential medication bias in the study presented was eliminated by (a) including treatment naïve patients (RRMS samples), by (b) including PPMS patients which have been off medication for at least three months prior to sampling, and by (c) excluding patients with known metabolic diseases in our clinical cohorts. Based on the given medication, it was not possible to build a significant PLS-DA model indicating that medication was no confounding factor in our identified marker profile. Moreover, analysis for our different PPMS, RRMS, and PD cohorts as well as corresponding controls was not biased as equal procedures for blood withdrawal, processing, storage, analytics, data acquisition, and analysis were applied to all samples. Overall, these results indicate that sex, gender, and medication appear to have no effects as confounding factors on our PPMS marker profile. Differences in age appear to be disease specific. Even though, our selection of 20 significantly changed metabolites overlapping between both PPMS cohorts appears to be lower than expected by chance, the set of the top 25 metabolites in both PPMS models (i.e., a larger candidate set than the 20 metabolites selected for stricter reasons and now based on their weighted VIP scores alone) reaches significance $(p$-value $=0.0182)$. Thus, applying a multivariate prediction model (PLS-DA), the metabolites detected relevant in one cohort proved informative in the second cohort as well. 


\section{Pathway Alterations in PPMS}

Even though the main objective of our study was to identify novel metabolite-based biomarkers for PPMS diagnosis that change during disease course our study also informs on the pathophysiology of the disease by the identification of alterations in distinct metabolite pathways (Supplementary Figure S7). We identified reduced levels of five phosphatidylcholines (PC), four lysophosphatidylcholines (lysoPC), and one glycerophosphatidylcholine (GPC) in PPMS patients. PC lipids and a large range of phospholipids are represented in the majority of eukaryotic cellular membranes. The $\mathrm{PLA}_{2}$ superfamily of enzymes catalyzes the hydrolysis of $s n$-2 ester bonds of glycerophospholipids (including PCs), resulting in the production of free fatty acids and lysophospholipids (including lysoPCs; Murakami and Kudo, 2002). PLA 2 products including lysoPCs are involved in a multitude of downstream pathways, orchestrating signal transduction via second messenger generation, driving biosynthesis of inflammatory mediators, neurotransmitter release, cell growth, differentiation, and apoptosis (Farooqui, 2009). Evidence of a deregulation of $\mathrm{PLA}_{2}$ and its products in disease has accumulated over the last decades and has been reported for several diseases including primary neurodegenerative diseases, such as Alzheimer's disease (AD), PD, amyotrophic lateral sclerosis (ALS), ischemia, and spinal cord injury (Farooqui and Horrocks, 2006). Interestingly, our findings of reduced lysoPC and PC species are supported by previous reports of an altered phospholipid metabolism in serum of MS patients (Del Boccio et al., 2011). Since both concentration and activity of lipoprotein-associated phospholipase A2 (Lp-PLA $A_{2}$ ) are similar in plasma of PPMS patients and controls (Sternberg et al., 2012), an attractive hypothesis is a disease-specific functional impairment of brain-specific PLA PL $_{2}$ which could account for lysoPC depletion. Alternatively, lysoPC species are generated by the enzyme lecithin:cholesterol acyltransferase (LCAT), which catalyzes the transfer of a fatty acyl residue from the $s n-2$ position of phosphatidylcholine to the 3-beta-hydroxy group of cholesterol, resulting in the formation of cholesteryl esters. Early studies, however, have suggested an increased LCAT activity in plasma of progressive MS patients compared to $\mathrm{HC}$ which is contradictory to our findings (Andreoli et al., 1973).

Our untargeted metabolomics approach revealed some additional statistically significant changes in 2(R)-HOT and gamma-Linolenic acid, products of linoleic acid metabolism. These metabolites are essential for the production of several omega-3 fatty acids, which in turn inhibit the actions of arachidonic acid and its pro-inflammatory derivatives. A randomized, double-blind, placebo-controlled, proof-ofconcept clinical trial treating RRMS patients with omega-3 fatty acids supplementation has reached the primary endpoint (reduction in annual relapse rate; Pantzaris et al., 2013). These findings are in line with the hypothesis of a deregulated linoleic acid metabolism in MS patients.

Of note, a deregulation of (L)-tryptophan in cerebrospinal fluid (CSF) and plasma of MS patients was reported several times and is consistent with our findings (Monaco et al., 1979;
Rudzite et al., 1996; Sandyk, 1996; Cocco et al., 2016). (L)-tryptophan is a substrate in the kynurenine pathway which is of particular interest in neuroinflammatory diseases because it contributes to immune regulation and generates both neurotoxic and neuroprotective mediators. Evidence of the link between the kynurenine pathway and MS pathogenesis is already well established and targeting crucial enzymes in this pathway could be a future treatment option (Lovelace et al., 2016; Lim et al., 2017).

In addition, we found depleted levels of citrulline. Citrulline plays a role in arginine biosynthesis and is also generated by arginine conversion. Perturbations in protein citrullination, in particular in citrullination of myelin basic protein (MBP), have been reported in MS and enzymes responsible for arginine citrullination (peptidyl arginine deiminases, PADs) have been discussed as potential molecular targets for MS therapy (Yang et al., 2016). To what extend freely circulating citrulline in the peripheral blood mirrors aberrantly citrullinated proteins in the central nervous system remains speculative at this point.

\section{LysoPC(20:0) Declines Over PPMS Disease Course}

Notably, almost all metabolites that led to a discrimination between HC and PPMS patients showed decreased concentrations in the latter and were stable over the investigated time course (24 months). The lipid annotated as LysoPC(20:0) is a notable exception since it significantly declines over the 24-month disease course. In contrast, elevated levels of LysoPC(20:0) were found in RRMS, PD patients, and compared to their matched HCs indicating PPMS specificity. Taken together, the significantly altered metabolite species reported here could allow for their future diagnostic application soon after disease onset. Whether LysoPC(20:0) could serve as a surrogate marker for DD and/or for the extent of neurodegeneration remains to be explored in a prospective cohort, preferably also including a longitudinal HC study arm. Moreover, to further investigate LysoPC (20:0) as a potential progression marker, its levels should be fully quantified in PPMS patients and HC over time.

\section{CONCLUSION}

In conclusion, metabolic profiling of plasma was found to be a promising technology for non-invasive diagnosis of PPMS. The results of this study led to the identification of a potential marker panel for PPMS diagnosis, which is decreased in plasma concentration compared to HC, RRMS, and PD. This also provides insights into specifically altered glycerophospholipid and linoleic acid metabolisms in PPMS patients. In future studies, it will be necessary to investigate the discriminatory power of the reported potential PPMS marker(s) in specimen of equally well-characterized larger clinical cohorts. Our results open a new line of investigation to specifically monitor phosphatidylcholine species as potential biomarkers for disease diagnosis and disease course progression. 


\section{DATA AVAILABILITY STATEMENT}

\section{Restrictions Apply to the Datasets}

The patient-associated datasets of this study cannot be made publicly available because of the nature of the study (as stated in the ethical documentation of the Institute for Neuroimmunology und Multiple Sclerosis and the Neuro Biobank Tübingen). Requests to access the datasets should be directed to the corresponding author.

\section{AUTHOR'S NOTE}

Part of this work has been submitted for patent registration at the European Patent Organisation (EPO), No. 17159341.1.

\section{AUTHOR CONTRIBUTIONS}

DS and J-PS have analyzed and interpreted data, drafted/revised the manuscript for content, and have contributed to study concept and design. AW, SCR, KS, and DW have analyzed and interpreted data and have revised the manuscript for content. $\mathrm{BB}$ and $\mathrm{SH}$ have analyzed and interpreted data. SR and $\mathrm{CD}$ have analyzed data. SF, WM, DB, and $\mathrm{CH}$ have contributed to study concept and design. NS revised the manuscript for content and has contributed to study concept and design. MF and OP have interpreted data, drafted/revised the manuscript for content, and have contributed to study concept and design.

\section{FUNDING}

The study was supported by the Bundesministerium für Bildung und Forschung (BMBF, grants 16GW0082 and 16GW0103K to OP; 16GW0053 to MF and 16GW0054 to NS and DS). The Neuro-Biobank of the University of Tübingen, Germany, received additional support from the local University, the Hertie-Institut für klinische Hirnforschung (HIH), and the Deutsches Zentrum für Neurodegenerative Erkrankungen (DZNE).

\section{SUPPLEMENTARY MATERIAL}

The Supplementary Material for this article can be found online at: https://www.frontiersin.org/articles/10.3389/fnhum. 2018.00226/full\#supplementary-material

FIGURE S1 | Workflow and summary of metabolomic profiling. (A) General workflow in data analysis. (B) Pie chart illustrating the percentage of putatively identified metabolites from each metabolite class, classified according to KEGG, Lipidmaps and HMDB. In total 534 metabolites were analysed. (C) PLS-DA scores plot of all 534 metabolites in HC cohort A ( $n=13$ ) (black) and PPMS cohort $\mathrm{A}(n=13)$ (green). (D) PLS-DA scores plot 534 metabolites identified in the HC cohort B $(n=20)$ (black) and PPMS cohort B $(n=20)$ (blue). Data in $(\mathbf{C}, \mathbf{D})$ show clear a separation between PPMS patients and HC. Ellipses assume a multivariate Gaussian distribution (2 sigma).
FIGURE S2 | Extracted ion chromatograms of metabolites contributing to the specific PPMS signature.

FIGURE S3 | Linear model fit of PPMS marker levels and age of all analyzed individuals in PPMS cohorts A and B. Utilized linear model to fit correlation between PPMS marker levels and ageing. Black: Linear model for all data points, light red: linear model for $\mathrm{HC}$ cohort $\mathrm{A}$, green: linear model for $\mathrm{HC}$ cohort $\mathrm{B}$, blue: linear model for PPMS cohort A, purple: linear model for PPMS cohort B. Grey areas around the lines indicate the 0.95 confidence interval. Metabolites with significant $(p$-value $<0.05)$ age and level correlations are highlighted with a red rectangle.

FIGURE S4 | PPMS marker levels and sex dependency in all individuals analyzed. Utilized one way ANOVA with Tukey's post hoc test to compute differences between PPMS marker levels and sex in all groups. *Metabolites with significantly different levels between males and females ( $p$-value <0.05), **Metabolites with significantly different levels between males and females ( $p$-value $<0.01$ ).

FIGURE S5 | Linear model fit of PPMS marker levels and age of all individuals analyzed in the PD cohort. Utilized linear model to fit correlation between PPMS marker levels and ageing. Black: Linear model for all data points, green: linear model for HC, purple: linear model for PD. Grey areas around the lines indicate the 0.95 confidence interval. Metabolites with significant $(p$-value < 0.05) correlations between age and level are highlighted with a red rectangle.

FIGURE S6 | Linear model fit of PPMS marker levels and age of all analyzed individuals in the RRMS cohort. Utilized linear model to fit correlation between PPMS marker levels and ageing. Black: Linear model for all data points, green: linear model for HC, orange: linear model for RRMS. Grey areas around the lines indicate the 0.95 confidence interval. Metabolites with significant $(p$-value $<0.05)$ age and level correlations are highlighted in a red rectangle.

FIGURE S7 | Pathway analysis of identified changes in PPMS patients compared to healthy controls $(\mathrm{HC})$. Pathway information retrieved from KEGG. Significantly altered metabolites are highlighted in dark red rectangles including the corresponding KEGG ID. Grey font indicates involved enzymes, dashed grey line indicates additional reactions which are not displayed. The substrates and products of reactions are shown in grey rectangular boxes. Green boxes indicate possible cellular function of observed changes in each pathway, decreased levels of metabolites compared to $\mathrm{HC}$ are indicated by blue arrows. Possible products and substrates are only partially displayed. Lysophosphatidylcholines lysoPE(18:1), lysoPE(18:2), and lysoPE(22:4) without reported KEGG ID (proposed KEGG ID C00438).

TABLE S1 | Cohorts. Patient information from investigated RRMS, PPMS, PD cohorts including age and gender. A disease duration of 0 corresponds to less than 1 year (*.xlsx file).

TABLE S2 | Inter-cohort dependencies determined by chi-square test for gender and one-way ANOVA for age, EDSS and disease duration (DD). Comparisons with significant $p$-values $(p$-value $<0.05$ ) are highlighted in bold red.

TABLE S3 | MS/MS fragments used for PPMS marker identification. Identities confirmed by available authentic standards (validation level 1). MS/MS spectra were matched against online databases such as Metlin and MassBank (validation level 2) or against in silico fragmentation spectra (validation level 3) retrieved from Metfrag, CFM-ID and/or CSI:FingerID with precursor mass accuracy of $20 \mathrm{ppm}$ and fragment accuracy of $0.01 \mathrm{Da}$.

TABLE S4 | Plasma metabolites identified in PPMS cohorts A and B (*.xlsx file).

TABLE S5 | Model parameters for the tested models and their corresponding $\mathrm{p}$-values after sample-label permutation test.

TABLE S6 | Metabolites with significant changes between $\mathrm{HC}$ and PPMS patients (A and B cohorts) their corresponding AUC values and VIP score ranks for component 1 and 2. Univariate AUCs and 95\% Cl (500 bootstrapping) calculated using MetaboAnalyst.

TABLE S7 | PPMS marker in PPMS patients compared to RRMS and PD patients. First $p$-value is determined by one-way ANOVA with Tukey's post hoc with correction for multiple testing per metabolite. Metabolites with $p$-values 
$<0.05$ are highlighted in bold green. Second $p$-value has been corrected (FDR) for all comparison including all metabolites.

TABLE S8 | PPMS marker measured in RRMS, intensity levels in the RRMS cohort. $P$-values determined by Welch's $t$-test, $p$-value adjustment for multiple comparison using Benjamini and Hochberg (FDR) adjustment (*.xlsx file).

\section{REFERENCES}

Allen, F., Pon, A., Wilson, M., Greiner, R., and Wishart, D. (2014). CFM-ID: a web server for annotation, spectrum prediction and metabolite identification from tandem mass spectra. Nucleic Acids Res. 42, W94-W99. doi: 10.1093/nar/ gku436

Andreoli, V. M., Maffei, F., Tonon, G. C., and Zibetti, A. (1973). Significance of plasma lysolecithin in patients with multiple sclerosis: a longitudinal study. J. Neurol. Neurosurg. Psychiatry 36, 661-667. doi: 10.1136/jnnp.36.4.661

Antel, J., Antel, S., Caramanos, Z., Arnold, D. L., and Kuhlmann, T. (2012). Primary progressive multiple sclerosis: part of the MS disease spectrum or separate disease entity? Acta Neuropathol. 123, 627-638. doi: 10.1007/s00401-0120953-0

Benjamini, Y., and Hochberg, Y. (1995). Controlling the false discovery rate: a practical and powerful approach to multiple testing. J. R. Stat. Soc. Ser. B 57, 289-300.

Botas, A., Campbell, H. M., Han, X., and Maletic-Savatic, M. (2015). Metabolomics of neurodegenerative diseases. Int. Rev. Neurobiol. 122, 53-80. doi: 10.1016/bs. irn.2015.05.006

Cocco, E., Murgia, F., Lorefice, L., Barberini, L., Poddighe, S., Frau, J., et al. (2016). (1)H-NMR analysis provides a metabolomic profile of patients with multiple sclerosis. Neurol. Neuroimmunol. Neuroinflamm. 3:e185. doi: 10.1212/ NXI.0000000000000185

Contrepois, K., Jiang, L., and Snyder, M. (2015). Optimized analytical procedures for the untargeted metabolomic profiling of human urine and plasma by combining hydrophilic interaction (HILIC) and reverse-phase liquid chromatography (RPLC)-Mass spectrometry. Mol. Cell. Proteomics 14, 16841695. doi: $10.1074 / \mathrm{mcp} . \mathrm{M} 114.046508$

Creek, D. J., Jankevics, A., Breitling, R., Watson, D. G., Barrett, M. P., and Burgess, K. E. (2011). Toward global metabolomics analysis with hydrophilic interaction liquid chromatography-mass spectrometry: improved metabolite identification by retention time prediction. Anal. Chem. 83, 8703-8710. doi: 10.1021/ac2021823

Creek, D. J., Jankevics, A., Burgess, K. E., Breitling, R., and Barrett, M. P. (2012). IDEOM: an excel interface for analysis of LC-MS-based metabolomics data. Bioinformatics 28, 1048-1049. doi: 10.1093/bioinformatics/bts069

De Livera, A. M., and Bowne, J. B. (2014). Metabolomics: Analysis of Metabolomics Data. $R$ package version 0.1.4.

Del Boccio, P., Pieragostino, D., Di Ioia, M., Petrucci, F., Lugaresi, A., De Luca, G., et al. (2011). Lipidomic investigations for the characterization of circulating serum lipids in multiple sclerosis. J. Proteomics 74, 2826-2836. doi: 10.1016/j. jprot.2011.06.023

Duhrkop, K., Shen, H., Meusel, M., Rousu, J., and Bocker, S. (2015). Searching molecular structure databases with tandem mass spectra using CSI:FingerID. Proc. Natl. Acad. Sci. U.S.A. 112, 12580-12585. doi: 10.1073/pnas.15097 88112

Farooqui, A. A. (2009). Lipid mediators in the neural cell nucleus: their metabolism, signaling, and association with neurological disorders. Neuroscientist 15, 392-407. doi: 10.1177/1073858409337035

Farooqui, A. A., and Horrocks, L. A. (2006). Phospholipase A2-generated lipid mediators in the brain: the good, the bad, and the ugly. Neuroscientist 12, 245-260. doi: 10.1177/1073858405285923

Floegel, A., Drogan, D., Wang-Sattler, R., Prehn, C., Illig, T., Adamski, J., et al. (2011). Reliability of serum metabolite concentrations over a 4-month period using a targeted metabolomic approach. PLoS One 6:e21103. doi: 10.1371/ journal.pone.0021103

Fox, R. J., Thompson, A., Baker, D., Baneke, P., Brown, D., Browne, P., et al. (2012). Setting a research agenda for progressive multiple sclerosis: the International Collaborative on Progressive MS. Mult. Scler. 18, 1534-1540. doi: 10.1177/ 1352458512458169
TABLE S9 | PPMS marker measured in PD, intensity levels in the PD cohort. P-values determined by Welch's $t$-test, $p$-value adjustment for multiple comparison using Benjamini and Hochberg (FDR) adjustment (*.xlsx file).

TABLE S10 | Metabolite levels with significant changes between $\mathrm{HC}$ and PPMS patients measured in the longitudinal PPMS cohort (*.xlsx file).

Friese, M. A., Schattling, B., and Fugger, L. (2014). Mechanisms of neurodegeneration and axonal dysfunction in multiple sclerosis. Nat. Rev. Neurol. 10, 225-238. doi: 10.1038/nrneurol.2014.37

Hope, R. M. (2013). Rmisc R Package. Available at: https://cran.r-project.org/web/ packages/Rmisc/index.html

Hughes, A. J., Ben-Shlomo, Y., Daniel, S. E., and Lees, A. J. (1992). What features improve the accuracy of clinical diagnosis in Parkinson's disease: a clinicopathologic study. Neurology 42, 1142-1146. doi: 10.1212/WNL.42.6.1142

Katz Sand, I. (2015). Classification, diagnosis, and differential diagnosis of multiple sclerosis. Curr. Opin. Neurol. 28, 193-205. doi: 10.1097/WCO. 0000000000000206

Kirwan, J. A., Weber, R. J., Broadhurst, D. I., and Viant, M. R. (2014). Direct infusion mass spectrometry metabolomics dataset: a benchmark for data processing and quality control. Sci. Data 1:140012. doi: 10.1038/sdata. 2014.12

Koch, M., Kingwell, E., Rieckmann, P., and Tremlett, H. (2009). The natural history of primary progressive multiple sclerosis. Neurology 73, 1996-2002. doi: 10.1212/WNL.0b013e3181c5b47f

Kuhn, M., Wing, J., Weston, S., Williams, A., Keefer, C., Engelhardt, A., et al. (2016). Caret: Classification and Regression Training. R package version 6.0-68.

Le Cao, K. A., Gonzalez, I., and Dejean, S. (2009). integrOmics: an R package to unravel relationships between two omics datasets. Bioinformatics 25, 28552856. doi: 10.1093/bioinformatics/btp515

Le Cao, K. A., Gonzalez, I., Dejean, S., Rohart, F., Gautier, B., Monget, P., et al. (2015). mixOmics: Omics Data Integration Project. R package version 5.2.0.

Libiseller, G., Dvorzak, M., Kleb, U., Gander, E., Eisenberg, T., Madeo, F., et al. (2015). IPO: a tool for automated optimization of XCMS parameters. BMC Bioinformatics 16:118. doi: 10.1186/s12859-015-0562-8

Lim, C. K., Bilgin, A., Lovejoy, D. B., Tan, V., Bustamante, S., Taylor, B. V., et al. (2017). Kynurenine pathway metabolomics predicts and provides mechanistic insight into multiple sclerosis progression. Sci. Rep. 7:41473. doi: 10.1038/ srep41473

Lovelace, M. D., Varney, B., Sundaram, G., Franco, N. F., Ng, M. L., Pai, S., et al. (2016). Current evidence for a role of the kynurenine pathway of tryptophan metabolism in multiple sclerosis. Front. Immunol. 7:246. doi: 10.3389/fimmu. 2016.00246

Monaco, F., Fumero, S., Mondino, A., and Mutani, R. (1979). Plasma and cerebrospinal fluid tryptophan in multiple sclerosis and degenerative diseases. J. Neurol. Neurosurg. Psychiatry 42, 640-641. doi: 10.1136/jnnp.42.7.640

Murakami, M., and Kudo, I. (2002). Phospholipase A2. J. Biochem. 131, 285-292. doi: 10.1093/oxfordjournals.jbchem.a003101

Ontaneda, D., Thompson, A. J., Fox, R. J., and Cohen, J. A. (2017). Progressive multiple sclerosis: prospects for disease therapy, repair, and restoration of function. Lancet 389, 1357-1366. doi: 10.1016/S0140-6736(16) 31320-4

Pantzaris, M. C., Loukaides, G. N., Ntzani, E. E., and Patrikios, I. S. (2013). A novel oral nutraceutical formula of omega-3 and omega- 6 fatty acids with vitamins (PLP10) in relapsing remitting multiple sclerosis: a randomised, double-blind, placebo-controlled proof-of-concept clinical trial. BMJ Open 3:e002170. doi: 10.1136/bmjopen-2012-002170

Patti, G. J., Yanes, O., and Siuzdak, G. (2012). Innovation: metabolomics: the apogee of the omics trilogy. Nat. Rev. Mol. Cell Biol. 13, 263-269. doi: 10.1038/nrm3314

Polman, C. H., Reingold, S. C., Banwell, B., Clanet, M., Cohen, J. A., Filippi, M., et al. (2011). Diagnostic criteria for multiple sclerosis: 2010 revisions to the McDonald criteria. Ann. Neurol. 69, 292-302. doi: 10.1002/ana. 22366

Redestig, H., Fukushima, A., Stenlund, H., Moritz, T., Arita, M., Saito, K., et al. (2009). Compensation for systematic cross-contribution improves normalization of mass spectrometry based metabolomics data. Anal. Chem. 81, 7974-7980. doi: 10.1021/ac901143w 
Rudzite, V., Berzinsh, J., Grivane, I., Fuchs, D., Baier-Bitterlich, G., and Wachter, H. (1996). Serum tryptophan, kynurenine, and neopterin in patients with GuillainBarre-syndrome (GBS) and multiple sclerosis (MS). Adv. Exp. Med. Biol. 398, 183-187. doi: 10.1007/978-1-4613-0381-7_30

Ruttkies, C., Schymanski, E. L., Wolf, S., Hollender, J., and Neumann, S. (2016). MetFrag relaunched: incorporating strategies beyond in silico fragmentation. J. Cheminform. 8:3. doi: 10.1186/s13321-016-0115-9

Sandyk, R. (1996). Tryptophan availability and the susceptibility to stress in multiple sclerosis: a hypothesis. Int. J. Neurosci. 86, 47-53. doi: 10.3109/ 00207459608986697

Scheltema, R. A., Jankevics, A., Jansen, R. C., Swertz, M. A., and Breitling, R. (2011). PeakML/mzMatch: a file format, Java library, R library, and tool-chain for mass spectrometry data analysis. Anal. Chem. 83, 2786-2793. doi: 10.1021/ac20 00994

Signori, A., Izquierdo, G., Lugaresi, A., Hupperts, R., Grand'maison, F., Sola, P., et al. (2017). Long-term disability trajectories in primary progressive MS patients: a latent class growth analysis. Mult. Scler. 24, 642-652. doi: 10.1177/ 1352458517703800

Sing, T., Sander, O., Beerenwinkel, N., and Lengauer, T. (2005). ROCR: visualizing classifier performance in R. Bioinformatics 21, 3940-3941. doi: 10.1093/ bioinformatics/bti623

Smith, C. A., Want, E. J., O'maille, G., Abagyan, R., and Siuzdak, G. (2006). XCMS: processing mass spectrometry data for metabolite profiling using nonlinear peak alignment, matching, and identification. Anal. Chem. 78, 779787. doi: 10.1021/ac051437y

Stellmann, J. P., Neuhaus, A., Lederer, C., Daumer, M., and Heesen, C. (2014). Validating predictors of disease progression in a large cohort of primaryprogressive multiple sclerosis based on a systematic literature review. PLoS One 9:e92761. doi: 10.1371/journal.pone.0092761

Sternberg, Z., Drake, A., Sternberg, D. S., Benedict, R. H., Li, F., Hojnacki, D., et al. (2012). Lp-PLA2: inflammatory biomarker of vascular risk in multiple sclerosis. J. Clin. Immunol. 32, 497-504. doi: 10.1007/s10875-011-9642-3

Stoessel, D., Schulte, C., Teixeira Dos Santos, M. C., Scheller, D., RebolloMesa, I., Deuschle, C., et al. (2018). Promising metabolite profiles in the plasma and CSF of early clinical Parkinson's Disease. Front. Aging Neurosci. 10:51. doi: 10.3389/fnagi.2018.00051

Sysi-Aho, M., Katajamaa, M., Yetukuri, L., and Oresic, M. (2007). Normalization method for metabolomics data using optimal selection of multiple internal standards. BMC Bioinformatics 8:93. doi: 10.1186/1471-2105-8-93
Thevenot, E. A., Roux, A., Xu, Y., Ezan, E., and Junot, C. (2015). Analysis of the human adult urinary metabolome variations with age, body mass index, and gender by implementing a comprehensive workflow for univariate and opls statistical analyses. J. Proteome Res. 14, 3322-3335. doi: 10.1021/acs.jproteome. 5b00354

Tremlett, H., Paty, D., and Devonshire, V. (2005). The natural history of primary progressive MS in British Columbia. Can. Neurol. 65, 1919-1923. doi: 10.1212/ 01.wnl.0000188880.17038.1d

Voskuhl, R. R., and Gold, S. M. (2012). Sex-related factors in multiple sclerosis susceptibility and progression. Nat. Rev. Neurol. 8, 255-263. doi: 10.1038/ nrneurol.2012.43

Want, E. J., Masson, P., Michopoulos, F., Wilson, I. D., Theodoridis, G., Plumb, R. S., et al. (2013). Global metabolic profiling of animal and human tissues via UPLC-MS. Nat. Protoc. 8, 17-32. doi: 10.1038/nprot.2012.135

Wishart, D. S., Jewison, T., Guo, A. C., Wilson, M., Knox, C., Liu, Y., et al. (2013). HMDB 3.0-The Human Metabolome Database in 2013. Nucleic Acids Res. 41, D801-D807. doi: 10.1093/nar/gks1065

Xia, J., Sinelnikov, I. V., Han, B., and Wishart, D. S. (2015). MetaboAnalyst 3.0making metabolomics more meaningful. Nucleic Acids Res. 43, W251-W257. doi: 10.1093/nar/gkv380

Xiao, J. F., Zhou, B., and Ressom, H. W. (2012). Metabolite identification and quantitation in LC-MS/MS-based metabolomics. Trends Analyt. Chem. 32, 1-14. doi: 10.1016/j.trac.2011.08.009

Yang, L., Tan, D., and Piao, H. (2016). Myelin basic protein citrullination in multiple sclerosis: a potential therapeutic target for the pathology. Neurochem. Res. 41, 1845-1856. doi: 10.1007/s11064-016-1920-2

Conflict of Interest Statement: The authors declare that the research was conducted in the absence of any commercial or financial relationships that could be construed as a potential conflict of interest.

Copyright (C) 2018 Stoessel, Stellmann, Willing, Behrens, Rosenkranz, Hodecker, Stürner, Reinhardt, Fleischer, Deuschle, Maetzler, Berg, Heesen, Walther, Schauer, Friese and Pless. This is an open-access article distributed under the terms of the Creative Commons Attribution License (CC BY). The use, distribution or reproduction in other forums is permitted, provided the original author(s) and the copyright owner are credited and that the original publication in this journal is cited, in accordance with accepted academic practice. No use, distribution or reproduction is permitted which does not comply with these terms. 